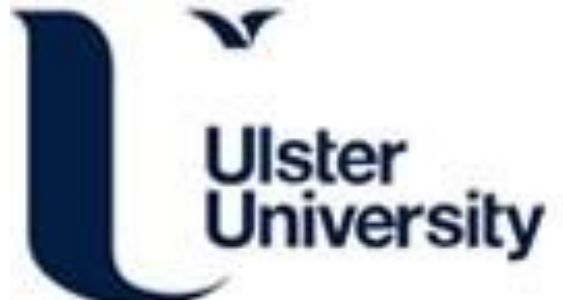

\section{Assessing possible DSM-5 ASD subtypes in a sample of victims meeting caseness for DSM-5 ASD based on self-report following multiple forms of traumatic exposure}

Hansen, M., Armour, C., Wang, L., Elklit, A., \& Bryant, R. A. (2015). Assessing possible DSM-5 ASD subtypes in a sample of victims meeting caseness for DSM-5 ASD based on self-report following multiple forms of traumatic exposure. Journal of Anxiety Disorders, 31, 84-89. https://doi.org/10.1016/j.janxdis.2015.02.005

Link to publication record in Ulster University Research Portal

Published in:

Journal of Anxiety Disorders

Publication Status:

Published (in print/issue): 01/01/2015

DOI:

10.1016/j.janxdis.2015.02.005

\section{Document Version}

Author Accepted version

\section{General rights}

Copyright for the publications made accessible via Ulster University's Research Portal is retained by the author(s) and / or other copyright owners and it is a condition of accessing these publications that users recognise and abide by the legal requirements associated with these rights.

\section{Take down policy}

The Research Portal is Ulster University's institutional repository that provides access to Ulster's research outputs. Every effort has been made to ensure that content in the Research Portal does not infringe any person's rights, or applicable UK laws. If you discover content in the Research Portal that you believe breaches copyright or violates any law, please contact pure-support@ulster.ac.uk. 
Running head: LPA ASD ACROSS MULTIPLE TRAUMATIC EXPOSURE

Assessing possible DSM-5 ASD subtypes in a sample of victims meeting caseness for DSM-5 ASD based on self-report following multiple forms of traumatic exposure

\author{
Maj Hansen, Ph. D. ${ }^{a}$ \\ Cherie Armour, Ph. $\mathrm{D}^{\mathrm{b}}$. \\ Li Wang Ph. $\mathrm{D}^{\mathrm{c}}$. \\ Ask Elklit, Msc. ${ }^{a}$ \\ Richard A. Bryant, Ph. D. ${ }^{\mathrm{d}}$
}

${ }^{a}$ National Centre for Psychotraumatology, Institute for Psychology, University of Southern Denmark;

Odense, Denmark

${ }^{\mathrm{b}}$ School of Psychology and Psychology Research Institute, University of Ulster, Coleraine, Northern Ireland, UK

${ }^{c}$ Key Laboratory of Mental Health, Institute of Psychology, Chinese Academy of Sciences, Beijing, China

${ }^{\mathrm{d}}$ School of Psychology, University of New South Wales, NSW, Australia

Category: research article

Correspondence concerning this article should be addressed to Maj Hansen, National Centre for

Psychotraumatology, Institute for Psychology, University of Southern Denmark, Campusvej 55, 5230

Odense M, Denmark. E-mail: mhansen@ health.sdu.dk, Phone: 0045-65502303 


\begin{abstract}
Acute Stress Disorder (ASD) was introduced into the DSM-IV to recognize early traumatic responses and as a precursor of PTSD. Although the diagnostic criteria for ASD were altered and structured more similarly to the PTSD definition in DSM-5, only the PTSD diagnosis includes a dissociative subtype. Emerging research has indicated that there also appears to be a highly symptomatic subtype for ASD. However, the specific nature of the subtype is currently unclear. The present study investigates the possible presence of ASD subtypes in a mixed sample of victims meeting caseness for DSM-5 ASD based on self-report following four different types of traumatic exposure $(N=472)$. The results of latent profile analysis revealed a five class solution. The highly symptomatic class was marked by high endorsement on avoidance and dissociation compared to the other classes. Findings are discussed in regard to its clinical implications including the implications for the pending the ICD-11 and the recently released DSM-5.
\end{abstract}

Keywords: acute stress disorder, latent profile analysis, ASD subtypes, DSM-5, ICD-11. 


\section{Introduction}

The acute stress disorder (ASD) diagnosis was introduced to the Diagnostic and Statistical Manual of Mental Disorders $4^{\text {th }}$ edition (DSM-IV) two decades ago, and ASD has subsequently been revised in the DSM-5 (DSM-IV; APA, 1994; 2013). The ASD diagnosis was originally introduced to recognize acute posttraumatic stress symptoms and as a way of identifying victims at risk of developing posttraumatic stress disorder (PTSD; APA, 1994). Notably, the idea that ASD is a precursor to PTSD, has been abandoned in the DSM-5 based on research which has repeatedly shown mixed results in relation to the capacity of the ASD diagnosis to predict the PTSD diagnosis (Bryant, 2011). One of the curious developments in DSM-5 was that ASD lost the emphasis on dissociative symptoms that initially distinguished it from PTSD in DSM-IV, and at the same time DSM-5 introduced a dissociative subtype of PTSD. In both the DSM-IV and the DSM-5, the diagnostic criteria of ASD and PTSD describe symptoms of intrusion, avoidance, and arousal. In the DSM-IV the main difference between the two diagnoses (besides the duration criterion) was that the DSM-IV ASD diagnosis required the presence of dissociative symptoms, whereas the DSM-IV PTSD diagnosis did not. The DSM-IV defines peritraumatic dissociation as a subjective feeling of emotional numbness, detachment from others, reduced responsiveness to one's surroundings, depersonalization, and derealization during the traumatic exposure (APA, 1994). Dissociation has been associated with both acute and long-term posttraumatic stress symptoms across numerous forms of traumatic exposure (c.f. Breh \& Seidler, 2007; Cardeña \& Carlson, 2011; Ozer, Best, Lipsey, \& Weiss, 2003). The importance of dissociation in relation to ASD has been reduced in the DSM-5 compared to the DSM-IV, since the DSM-5 ASD diagnosis no longer requires the presence of a specific number of dissociative symptoms, rather just the presence of 9 out of a total of 14 symptoms (i.e. those belonging to the five categories of intrusion, arousal, negative mood, avoidance, and dissociation). This change was made on the basis of increasing 
evidence that peritraumatic dissociation does not adequately predict PTSD with sufficient sensitivity and specificity (Bryant, 2011). The first formal recognition of dissociative presentations in longer-term traumatic responding occurred in DSM-5 because of initial evidence that PTSD patients with dissociative tendencies had distinctive neural and behavioral characteristics (Felmingham et al., 2008; Lanius et al., 2010).

Recently, several studies using latent profile analysis (LPA) have identified a number of PTSD subtypes including the dissociative subtype (cf. Armour, Elklit, Lauterbach, \& Elhai, 2014a; Armour, Karstoft, \& Richardson, 2014b; Wolf et al., 2012a; Wolf et al., 2012b). As argued by Armour and Hansen (2015), due to the close similarities in the diagnostic criteria of both disorders, severity and latent structure (c.f. Classen, Koopman, Hales \& Spiegel, 1998; Hansen, Armour, \& Elklit, 2012; Hansen \& Elklit, 2013; Wang et al., 2012) as well as two reviews of ASD (Cardeña \& Carlson, 2011; Isserlin, Zerach, \& Solomon, 2008) and the proposed ICD-11 recommendation for ASD symptoms (WHO, 2014), it is possible that a dissociative subtype may also be apparent for ASD. Furthermore, to the best of our knowledge only two studies have investigated the latent profile of ASD (Shevlin, Hyland \& Elklit, 2014; Armour \& Hansen, 2015). These two studies, combined with a limited body of research, suggest that alternative ASD subtypes are also likely to exist.

Using LPA on the mean scores of the four ASD DSM-IV symptom clusters, Shevlin, Hyland and Elklit (2014) found that the latent profiles of ASD in Danish victims of rape $(N=471)$ differ both quantitatively and qualitatively. The results of the LPA revealed four classes: high mean scores on all four ASD symptom clusters, low mean scores on all four DSM-IV ASD symptom clusters, and two intermediate classes. The two intermediate classes were similar in the mean number of dissociation and re-experiencing symptoms, but one intermediate class had a higher number of arousal and lower number of avoidance symptoms compared to the other intermediate class. Armour and Hansen (2015) 
used LPA on the 19 individual symptoms of DSM-5 ASD and identified four different classes in Danish victims of bank robbery $(N=450)$ : a highly symptomatic class, a class with moderate intrusion and high endorsement on the remaining items, a class characterized by low scores on most items except moderate to low on arousal, and a low symptomatic class. Although, the studies are not directly comparable due to different analytical approaches, combined the two studies suggest that ASD is not simply experienced by quantitative differences in severity, but instead qualitatively different profiles may also to exist. These profiles may also differ following different forms of traumatic exposure. Both studies were based on Danish victims of interpersonal violence with ASD prevalence rates of $68.8 \%$ according to the DSM-IV in rape victims and $10 \%$ according to the DSM-5 in bank robbery victims. As argued by Hansen et al. (2012) it is possible that the latent structure of ASD may differ in clinical samples compared to mixed samples. Thus, it is important to investigate the latent profile of ASD symptoms in clinical populations. At the same time, it is also possible that different subtypes of ASD exist across traumatic exposures and thus it is important to investigate the latent structure of ASD in a heterogeneous trauma sample. The existence of ASD subtypes may help to explain the mixed results regarding the capacity of ASD to predict PTSD across traumatic exposure and the confirmatory factor analytic studies failing to support the DSM-5 one-factor structure of ASD symptoms. Indeed, the PTSD latent factor structure is found to differ in veterans with and without PTSD (Biehn, Elhai, Fine, Seligman, \& Richardson, 2012). Thus, the present study investigated the latent profile of ASD symptoms in a sample of victims of different traumatic exposure meeting caseness for DSM-5 ASD based on self-report. Based on the limited research we hypothesized that different ASD subtypes would exist which are both quantitatively and qualitatively different. More specifically, we expected to find a highly symptomatic class and intermediate ASD severity subgroups. We did not expect to find a low symptomatic group as we were assessing ASD subtypes in a sample meeting caseness for DSM-5 ASD 
based on self-report and we were uncertain about the specific number of intermediate classes. Although, previous research has reached different conclusions in regard to ASD subtypes (i.e. dissociative or intrusive), we expected that we may uncover a dissociative ASD subtype given the highly symptomatic nature of the participants in the current study.

\section{Method}

\section{Participants}

Five separate samples were combined for the present study.

\section{Sample 1. - Victims of rape.}

Data was provided by a larger, ongoing, longitudinal study of rape victims who presented at a center for victims of rape at the University Hospital, Aarhus, Denmark. A total of 658 were administered the Acute Stress Disorder Scale (ASDS) two to three weeks after the sexual assault. However, 22 cases were missing $>20 \%$ and thus were excluded from the analysis, leaving an effective sample size of 636. For further details about the study please see an earlier version of this sample in Armour, Elklit and Shevlin (2011b).

\section{Sample 2 - Victims of bank robbery.}

The second sample comprised 152 bank employees exposed to bank robberies committed in Denmark between September 2008 and March 2010. Participants were recruited through a network of crisis intervention specialists contracted with bank organizations in Denmark and administered the ASDS one week after the robbery. However, three cases were missing $>20 \%$ and thus were excluded from the analysis, leaving an effective sample size of 149. For further details about the study, see Hansen and Elklit (2011).

\section{Sample 3 - Victims of bank robbery.}


Data was provided from a national study of bank robberies committed in Denmark from April 2010 to April 2011. The study was conducted in collaboration between the Danish Bankers Association, all Danish Banks, and the University Southern of Denmark. A total of 450 participants were administered the ASDS one week after the robbery. There were no cases with missing $>20 \%$. For further details about the study, see Hansen et al. (2012).

\section{Sample 4 - Victims of earthquake.}

The fourth sample comprised Chinese victims of earthquake collected 12-15 days after an earthquake measuring 5.8 magnitudes on the Richter scale occurred in 2011 in Yingjiang County, Yunnan province, People's Republic of China. A total of 358 victims filled out the questionnaire. However, eight cases were missing $>20 \%$ and thus were excluded from the analysis, leaving an effective sample size of 350. For further details about the study, see Wang et al. (2012).

\section{Sample 5 - violence at work}

Data was provided by a larger, ongoing, longitudinal study of employees at different Danish psychiatric departments, who reported a claim after exposure to either physical or psychological violence at work (e.g. beaten or threatened by patients). The study was conducted in collaboration between the University Southern of Denmark, the Occupational Health Clinic at Herning Hospital, and the Psychiatric Department of Southern Denmark. Three hundred and seven participants were administered the ASDS within a month of the reported incident. However, 2 cases were missing $>20 \%$ and thus were excluded from the analysis, leaving an effective sample size of 305.

\section{Full combined sample}

As shown in table 1, 359 victims of rape, 23 victims of bank robbery, 45 victims of bank robbery, 26 victims of earthquake, and 19 victims of violence at work met caseness for DSM-5 ASD based on 
self-report (please refer to the measure section for further details). Thus, the full combined sample had a total of 472 participants meeting caseness for DSM-5 ASD based on self-report.

[Please insert table 1]

\section{Measure}

The DSM-5ASD symptoms were assessed using the ASDS in all samples (Bryant, Moulds \& Guthrie, 2000). The ASDS is a 19 item self-report scale originally developed to assess the four DSM-IV ASD symptom clusters. However, as argued by Armour and Hansen (2015) the ASDS items bare close resemblance to the DSM-5 ASD symptoms and can be used to measure DSM-5 ASD. This is with one exception as the ASDS does not fully assess the B5 symptom for negative mood. More specifically, the DSM-5 defines the B5 symptom as a persistent inability to experience positive emotions, whereas the ASDS assess numbing in general and not solely in relation to positive emotions. Questions are answered on a five-point Likert scale $(1=$ not at all, $5=$ very much $)$. The participants met caseness for DSM-5 ASD based on self-report if they endorsed at least nine out of the 14 described symptoms; all indicated by item scores $\geq 3$ on the ASDS. Previous studies have reported good reliability for the ASDS total score (Cronbach's $\alpha=.76-.96$, Hansen et al., 2012). The reliability coefficient in the present study for the full combined sample was satisfactory (total scale $=.93$ ).

\section{Data analysis}

LPA, an exploratory modeling procedure, was conducted with Mplus version 7 (Muthén \& Muthén, 2012). LPA is a statistical method used to identify homogenous groups or classes from multivariate data. Details pertaining to the method of LPA can be found across several studies (cf. DiStefano \& Kamphaus, 2006; Lubke \& Muthén, 2005). Models including 19 indicators of ASD, of 
between 2 to 6 latent classes were specified and estimated in the present study. The models were estimated using robust maximum likelihood (Yuan \& Bentler, 2000). The relative fit of the models were compared using three established fit indices; the Akaike information criterion (AIC), the Bayesian information criteria (BIC), and the sample size adjusted BIC. Better fitting models are indicated by lower values. In addition, models were also compared based on the Lo-Mendell-Rubin loglikelihood adjusted ratio test (LMR-A) and the bootstrapped likelihood ratio test (BLRT). The LMR-A compares $\mathrm{k}$ and $\mathrm{k}-1$ latent class models. A non-significant LMR-A indicates that a model with one less class provides superior fit. Entropy values approaching 1 indicate clearer classification across classes. Entropy values $>.8$ are preferred.

\section{Results}

Table 1 shows sample characteristics of the five distinct samples and the total sample including the mean scores on the ASDS total score and the five symptom categories.

\section{Missing Data}

The percentages of missing values were low (.00-9.5 \%) across the different datasets. Thus, the Expectation Maximization algorithm was used for the imputation of missing values.

\section{Latent Profile Analysis (LPA)}

The resultant fit indices across the 2-6 latent class models are presented in Table 2. Each increment in the number of latent classes' saw a decrease in AIC, BIC, and SSABIC values with the exception of the BIC which saw an increase from the 5- to 6-class model. Continual decreases in AIC, BIC, SSBIC fit statistics are common, however, DiStefano and Kamphaus (2006) suggested that the degree of decrease should be calculated and when a notable tailing off occurs. When there are only 
marginal differences in values between classes the addition of one extra class is deemed unnecessary. In the present study this occurred for the AIC and SSABIC between latent class models of 5- and 6classes. Combined with the increase in the BIC value from the 5- to 6-class solution the 5 class model is deemed optimal based on these three fit indices.

[Please insert table 2]

The LMR-A, failed to reach significance from the 3-class model onwards. However, when assessing the BLMR-A all latent models reached significance. Entropy values for the 2-, 3-, and 4-class models fell below the optimal entropy value of $>0.8$ suggesting less clarity in the classification of individuals across classes. However the entropy value in the 5- and 6- class models exceeded .8 suggesting clearer classification. Taking a balance of these fit indices, parsimony, and substantive meaning into consideration we rejected the 2-, 3-, 4- and 6-class models in favor of the 5-class model.

In the optimal model, class 1 comprised $10.1 \%$ of the sample whereas classes $2,3,4$, and 5 , comprised $17.6 \%, 32.6 \%, 22.7 \%$, and $16.8 \%$ of individuals respectively. Please see figure one for the profile plot of the 5-class model. Notable features of class 1 are that these individuals are reporting low levels of dissociation relative to alternative classes. Notable to class 2 is that these individuals are reporting low levels of avoidance relative to alternative classes. Individuals in class 3 report moderate symptomatology across all ASD factor with the exception of intrusion; which is low relative to alternative classes. Class 4 report elevates means of intrusion and arousal relative to other classes with the exception of class 5. Class 5 report the highest means across all ASD factor compared to all other classes. Of note however class 4 reports the highest means on 2 out of 5 intrusive items. 


\section{Discussion}

The present study is one of few studies investigating the latent structure of ASD with a focus on uncovering latent subgroups using LPA. To the best of our knowledge the present study is the first study to investigate the existence of possible ASD subtypes in a large heterogeneous trauma sample meeting caseness for DSM-5 ASD based on self-report. Based on the limited research we expected to find a highly symptomatic class and a number of intermediate ASD severity groups. Furthermore, we expected that the highly symptomatic ASD subtype would be of a dissociative nature as found in the PTSD research (c.f. Armour et al, 2014a; 2014b; Wolf et al., 2012a; 2012b).

The results of the LPA revealed that participants were optimally grouped into five latent classes: four intermediate ASD severity classes (i.e. class 1-4) and a highly symptomatic class (i.e. class 5). As expected from investigating profiles of ASD severity in a highly symptomatic sample, we did not find a distinct low symptomatic class as found by the two previous studies investigating latent subtypes of ASD in victims with and without ASD (Armour \& Hansen, 2015; Shevlin et al., 2014).The notable features of the intermediate classes in the present study compared to the other classes are that class 1 had the lowest levels of dissociation, class 2 the lowest levels of avoidance, class 3 the lowest levels of intrusion, and class 4 reported high levels of intrusion and arousal relative to the other classes with the exception of class 5. Thus, in accordance with ASD reviews (Cardeña \& Carlson. 2011; Isserlin et al., 2008), Shevlin et al. (2014) and Armour and Hansen (2015) we found that a variety of ASD subtypes do exist. Although, we did not find a low symptomatic group due to the clinical nature of our sample, the results are in accordance with previous research suggesting that latent ASD profiles differ both quantitatively and qualitatively. In contrast to Armour and Hansen (2015) and research in PTSD subtypes (Armour et al., 2014a; 2014b; Wolf et al., 2014a; 2014b), we did not find an ASD subtype marked by intrusion or marked only by dissociation. Instead, we found that the highly symptomatic 
profile was marked by higher scores on both dissociation and avoidance compared to the other classes. This suggests that the specific nature of the latent profiles of ASD symptoms may depend on whether symptoms are assessed in victims with or without ASD. Of note, the majority of the participants in the present study were female. This is expected in a highly symptomatic sample as female sex has been repeatedly found associated with higher levels of both ASD, dissociation, increased risk of PTSD, and higher prevalence of dissociative PTSD compared to males (c.f. Armour et al., 2011a; Bryant and Harvey, 2003; Tolin \& Foa, 2006; Wolf et al., 2012a; 2012b). One explanation for this may relate to sex differences in coping styles. Females are more likely to report the use of emotion focused coping styles than males and this type of coping style are found associated with PTSD severity regardless of sex (Tolin \& Foa, 2006). This may also explain why the highly symptomatic class in the present study is associated with reports of high dissociation compared to the Armour and Hansen (2015) study, where only $61 \%$ of the participants were female. However, the effect of the gender distribution is not likely to be large as female sex has not been found a significant risk factor for the highly symptomatic ASD subgroup (Armour \& Hansen, 2015) or the dissociative PTSD subtype (Armour et al., 2014b).

Although it was not our original hypothesis, it is not necessarily a surprising finding that the highly symptomatic class also had high avoidance when thinking about the nature of ASD. ASD takes place within the first month of traumatic exposure and in the present study symptoms were assessed between 1-3 weeks following traumatic exposure. Usually, it takes time for symptoms of avoidance to develop (O’Donnell, Elliott, Lau, \& Creamer, 2007) as victims will first need to develop avoidance strategies to avoid reminders of the traumatic exposure as they experience repeated episodes of intrusive and arousal symptoms. However, when victims are highly traumatized avoidance symptoms are likely to develop very fast in order to reduce distress and secure survival (Foa, Hembree, \& Rothbaum, 2007), which is likely to be the case with the highly symptomatic class in this study. This is 
in accordance with emotional processing theories assuming that fear structure networks are created in the memory when the traumatic exposure has acquired a meaning of threat and avoidant strategies are used to prevent experiencing distressing symptoms of intrusion and arousal (Foa, Steketee, \& Rothbaum, 1989). Paradoxically, the strategies have the opposite effect as they hinder habituation and modification of the traumatic memory network and thus have a preserving role on posttraumatic stress symptomatology. It is possible that avoidance is only related to the highly symptomatic ASD subgroup and not the highly symptomatic PTSD subgroup as avoidance symptoms have been found to increase and peak during the first three months of traumatic exposure (O’Donnell et al., 2007).

The results of the present study have several clinical implications. Combined with the two other studies of ASD subtypes (Armour \& Hansen, 2015; Shevlin et al., 2014), they suggest that different ASD subgroups may exist. Independently from the specific nature of these subgroups this underlines the need for clinicians to be aware of the possibility that ASD patients may present themselves with different prominent symptom profiles and thus may be in need of more specific tailored treatment plans. Indeed, as argued by Armour and Hansen (2015) one subgroup may respond differently to a particular treatment compared to other subgroups. In the present study, the results suggest that treatment of the highly symptomatic victims would benefit from focusing on relieving symptoms of avoidance and dissociative symptoms in the acute phrase following traumatic exposure. Additionally, the existence of possible ASD subgroups may help to explain the mixed results in relation to the capacity of ASD to predict PTSD as suggested by Shevlin et al. (2014). Unfortunately, the association between the highly symptomatic ASD subgroup found in the present study and the development of PTSD is unknown as it was not possible to assess PTSD in the combined sample due to the use of different PTSD measurements. Thus it is unknown whether the victims in the highly symptomatic ASD group are the same victims who go on to develop dissociative PTSD. However, the results do point to a 
possible explanation of the limited predictive power of ASD on PTSD. In a similar vein, the existence of ASD subgroups also points to a possible explanation for the lack of support found for the DSM-5 ASD structure in confirmatory factor analytic studies (Armour et al., 2011b; Hansen et al., 2012). The latent structure of ASD does not appear to be a simple one factor structure, but instead there appears to be subgroups as suggested by the proposed ICD-11 recommendations for acute traumatic stress symptoms (WHO, 2013). It is important that diagnostic systems are precise descriptions as they are used to guide screening and treatment. An imprecise description of ASD may mean that clinical work does not sufficiently target the right symptoms making treatment less efficient. At the same time, this also means that research may be guided in the wrong direction and thus fail to identify the correct risk factors; thus making it impossible to take efficient preventive actions.

\section{Limitations}

The present study is subjected to several limitations. The ASD symptoms were assessed using a self-report measures rather than clinical interviews. It is possible that the latent structure of ASD may differ depending on how it is assessed. At the same time, we did not fully assess the B5 criterion using the ASDS. Furthermore, ASD symptoms were assessed at different time-points across the different samples included in the present study. Although, the ASD diagnosis should apply for all ASD symptoms present within the first month of traumatic exposure, it cannot be ruled out that assessment time can have affected the results. Unfortunately, only the timeframes and not the specific assessment time-points were known. Thus, it was not possible to examine the effect of time in the current study. The majority of the sample were female which may question the generalizability of these results to all ASD patients. Furthermore, the majority of the sample were victims of rape (76\%). This means that the results may be more reflective of ASD subtypes in victims of rape and thus not generalize as well to 
victims of other forms of traumatic exposure than rape. However, it should be noted that the ASD diagnosis does not discriminate across trauma types and the proportion of victims of other forms of traumatic exposure than rape was large enough to have a significant impact on the results of the present study. Unfortunately, the combining of different datasets meant that we were only able to assess ASD profiles given the ASD measure was the common factor across datasets. We were therefore unable to assess predictors of ASD group membership or the risk related to the development of PTSD as a function of ASD group membership. Thus, currently the impact of ASD class membership on the development of PTSD is unknown.

\section{Conclusions}

The present study is the first to investigate latent profiles of the DSM-5 ASD diagnosis in a heterogeneous trauma population meeting caseness for DSM-5 ASD based on self-report. We found a five class solution with four intermediate classes and one highly symptomatic class. Contrary to our expectation the highly symptomatic class or the ASD subgroup class was primarily distinguished from the other all classes by both high endorsement on dissociation and avoidance. Thus, there appears to be a dissociative avoidant ASD subtype. Future studies should focus on replicating these results while including an assessment of the associated risk factors for highly symptomatic class membership as well as the connection between ASD subtypes and PTSD subtypes. Currently knowledge of the latent profiles of ASD is in its infancy but as knowledge is accumulated, future studies should also focus on exploring factor mixture models as they relate to the latent structure of ASD. This knowledge can have a potential impact on clinical practice and it can guide the development of more precise diagnostic description and thus a targeted treatment. 


\section{Acknowledgements}

The authors thank the Danish Bankers Association and the National Bank of Denmark for cofinancing the national bank robbery study. Furthermore, the authors would like to acknowledge Lars Peter Søndergaard Andersen, Hospital of Herning, Denmark for taking part in the design and recruitment of participants for the violence at work study. 
References

American Psychiatric Association (1994). Diagnostic and Statistical Manual of Mental Disorders, $4^{\text {th }} e d$. Washington DC: American Psychiatric Association.

American Psychiatric Association (2013). Diagnostic and Statistical Manual of Mental Disorders, $5^{\text {th }}$ ed. Washington DC: American Psychiatric Association.

Armour, C., Elhai, J.D., Layne, C.M., J.A., Shevlin, M., Duraković-Belko, E., Djapo, N., \& Pynoos, R.S. (2011a). Gender Differences in the Factor Structure of Posttraumatic Stress Disorder Symptoms in W ar-Exposed Adolescents. Journal of Anxiety Disorders 25, 604-611. doi: 10.1016/j.janxdis.2011.01.010

Armour, C., Elklit, A. \& Shevlin, M. (2011b). The latent structure of acute stress disorder. Psychological Trauma. doi: 10.1037/a0024848

Armour, C. \& Hansen, M. (2015). Assessing DSM-5 latent subtypes of acute stress disorder, dissociative or intrusive? Psychiatry Research 28, 476-483. doi: 10.1016/j.psychres.2014.11.063

Armour, C., Karstoft, K., \& Richardson, D. (2014). The co-occurrence of PTSD and dissociation: differentiating severe PTSD from dissociative-PTSD. Social Psychiatry and Psychiatric Epidemiology. doi: 10.1007/s00127-014-0819-y

Biehn, T., Elhai, J., Fine, T. H., Seligman, L. D., \& Richardson, J. D. (2012). PTSD factor structure differences between veterans with or without a PTSD diagnosis. Journal of Anxiety Disorders, 26, 480-485. http://dx.doi.org/10.1016/j.janxdis.2012.01.008

Breh, D. C., \& Seidler, G. H. (2007). Is peritraumatic dissociation a risk factor of PTSD? Journal of Trauma and Dissociation, 8, 53-69. doi: 10.1300/J229v08n01_04 
Bryant, R. A. (2011). Acute stress disorder as a predictor of posttraumatic stress disorder: a systematic review. Journal of Clinical Psychiatry, 72, 233-239. doi: 10.4088/JCP.09r05072blu

Bryant, R. A., \& Harvey, A. (2003). Gender differences in the relationship between acute stress disorder and posttraumatic stress disorder following motor vehicle accidents. Australian and New Zealand Journal of Psychiatry, 37, 226-229. doi:10.1046/j.1440-1614.2003.01130.x

Bryant, R. A., Moulds, M. L., \& Guthrie, R. M. (2000). Acute stress disorder scale. Psychological Assessment, 12, 61-68. doi: 10.1037//I040-3590.12.1.61

Cardeña, E., \& Carlson, E. (2011). Acute stress disorder revised. Annual Review of Clinical Psychology, 7, 245-267. doi: 10.1146/annurev-clinpsy-032210-104502

Classen, C., Koopman, C., Hales, R., \& Spiegel, D. (1998). Acute stress disorder as a predictor of posttraumatic stress symptoms. American Journal of Psychiatry, 155. 620-624. Retrieved from http://ajp.psychiatryonline.org

DiStefano, C., Kamphaus, R .W. (2006). Investigating Subtypes of Child Development: A Comparison of Cluster Analysis and Latent Class Cluster Analysis in Typology Creation. Educational and Psychological Measurement, 66, 778-794. doi: 10.1177/0013164405284033

Felmingham, K., Kemp, A. H., Williams, L., Falconer, E., Olivieri, G., Peduto, A., \& Bryant, R. (2008). Dissociative responses to conscious and non-conscious fear impact underlying brain function in post-traumatic stress disorder. Psychological Medince, 38, 1771-1780. doi: $10.1017 / \mathrm{S} 0033291708002742$

Foa, E. B., Hembree, E. A., \& Rothbaum, B. O. (2007). Prolonged exposure therapy for PTSD: Emotional processing of traumatic experiences. New York, NY: Oxford University Press. 
Foa, E. B., Steketee, G., \& Rothbaum, B. O. (1989). Behavioral/cognitive conceptualizations of posttraumatic stress disorder. Behavior Therapy, 20, 155-176.

Hansen, M., Armour, C., \& Elklit, A. (2012). Assessing a dysphoric arousal model of acute stress disorder symptoms in a clinical sample of rape and bank robbery victims. European Journal of Psychotraumatology, 3. doi:10.3402/ejpt.v3i0.18201

Hansen, M., \& Elklit, A. (2011). Predictors of acute stress disorder in response to bank robbery. European Journal of Psychotraumatology, 2. doi: 10.3402)ejpt.v2i0.5864

Hansen, M., \& Elklit, A. (2013). Does ASD predict PTSD following bank robbery? Journal of Interpersonal Violence, 28, 25-44. doi: 10.1177/0886260512448848.

Isserlin, L., Zerach, G., \& Solomon, Z. (2008). Acute stress responses: a review and synthesis of ASD, ASR, and CSR. American Journal of Orthopsychiatry 78:423-29. doi: 10.1037/a(x)14304

Lanius, R. A., Vermetten, E., Loewenstein, R. J., Brand, B., Schmahl, C., Bremner, J. D., \& Spiegel, D. (2010). Emotion Modulation in PTSD: Clinical and Neurobiological Evidence for a Dissociative Subtype. American Journal of Psychiatry, 167, 640-647.

Lubke, G. H. \& Muthén, B. (2005). Investigating population heterogeneity with factor mixture models. Psychological Methods, 10, 21-39. doi: 10.1037/1082-989X.10.1.21

Muthén L., \& Muthén, B., 2012. Mplus User Guide. Version 7. Los Angeles: Statmodel

O’Donnell, M., Elliott, P., Lau, W., \& Creamer, M. (2007). PTSD symptom trajectories: From early to chronic response. Behaviour Research and Therapy, 45, 601-606. doi:10.1016/j.brat.2006.03.015 
Ozer, E. J., Best, S. R., Lipsey, T. L., \& Weiss, D. S. (2003). Predictors of posttraumatic stress disorder and symptoms in Adults: A meta-Analysis. Psychological Bulletin, 129, 52-73. doi: 10.1037/0033-2909.129.1.52

Shevlin, M., Hyland, P., \& Elklit, A. (2014). Different profiles of acute stress disorder differentially predict posttraumatic stress disorder in a large sample of female victims of sexual trauma. Psychological Assessment. doi: 10.1037/a0037272

Tolin, D. F., \& Foa, E. (2006). Sex differences in trauma and posttraumatic stress disorder:A quantitative review of 25 years of research. Psychological Bulletin, 132, 959-992. doi: 10.1037/0033-2909.132.6.959

Wang, R., Wang, L., Zhang, J., Liu, Z., \& Wu, K. (2012). The structure of acute stress disorder among Chinese adults exposed to an earthquake: Is dysphoric arousal a unique construct of acute posttraumatic responses? Scandinavian Journal of Psychology, 53, 430-436. doi: 10.1111/j.14679450.2012 .009

WHO (World Health Organization) (2013). WHO guidelines on conditions specifically related to stress. Retrieved October 21, 2014 from http://www.who.int/mental_health/emergencies/stress_guidelines/en/

Wolf, E. J., Lunney, A., Miller, M. W., Resick, P. A., Friedman, M. J., \& Schnurr, P.P. (2012). The dissociative subtype of PTSD: A replication and extension. Depression and Anxiety, 29, 679688. doi: $10.1002 /$ da.21946

Wolf, E. J., Miller, M. W., Reardon, A. F., Ryabchenko, K. A., Castillo, D., \& Freund, R.(2012). A latent class analysis of dissociation and posttraumatic stress disorder evidence for a dissociative subtype. Archives of General Psychiatry, 69, 698-705. 
Yuan K. H., \& Bentler, P. M. (2000). Three likelihood-based methods for mean and covariance structure analysis with non-normal missing data. Sociological Methodology, 165-200. doi: $10.1111 / 0081-1750.00078$ 